\section{Liberté, égalité, fraternité (et santé)}

\author{
E. Taverna
}

Das vierte Treffen auf dem Weg zu einer nationalen Gesundheitspolitik vereinigt Regierungsräte, Chefbeamte, Ressortleiter, Zentralsekretäre, Geschäftsführer und Direktoren kantonaler und bundesamtlicher Stellen. Gesucht werden im Institut des Brückenbauers Gottlieb Duttweiler «die bestimmenden Faktoren der Gesundheit und ihre Bedeutung für die Gesundheitspolitik». Bundesrätin Ruth Dreifuss stellt fest, dass «die Götter in Weiss» nicht die Garanten unserer Gesundheit seien, und fragt sich, ob die jährlich 40 Milliarden Franken für die medizinische Versorgung wirklich die beste Investition sind.

\section{Die alte und neue Armut}

Das einleitende Referat hält Prof. Dr. med. Jean Daniel Reinhorn: "La progression de la précarité en France et ses effets sur la santé»; ein Bericht, der 1998 im Auftrag des Hochkommissariats für öffentliche Gesundheit verfasst wurde. Gemeint ist die zunehmende Gefährdung von 20 bis 25\% der Bevölkerung, von 12 bis 15 Millionen Menschen, die in ihrer psychischen und physischen Existenz bedroht sind: Kinder, die vorzeitig die Schule verlassen, unqualifizierte Jugendliche, Arbeitslose, die "working poors" und Alleinerziehende mit niedrigem Einkommen. Der Bericht nennt Ausgrenzung, Verunsicherung, fehlende Perspektiven, Mangel und Armut als Ursachen von Krankheit und Gewalt, die letztlich den nationalen Zusammenhalt bedrohen. Gefordert werden in erster Priorität menschenwürdige Arbeitsplätze, sichere und stabile Schulverhältnisse, das Recht auf anständige Behausung und gute Sozialversicherungen. Das entspricht auch den Leitlinien der Schweizerischen Gesellschaft für Prävention und Gesundheitswesen für eine "Gesundheitsförderliche Gesamtpolitik", die denselben Determinanten für Gesundheit noch eine gute Ernährung und ein stabiles Ökosystem beifügen. Reinhorn fordert eine "neue Kultur", die aufgrund sozio-ökonomischer Fakten Politik und Gesellschaft zu mehr Solidarität verpflichtet.

Was zahlreiche Studien für Europa und Neuseeland nachweisen, ist auch für die Schweiz belegt. In den letzten 10 Jahren driften arm und reich zunehmend auseinander. Die Zahl der Einelternfamilien hat sich verdoppelt und immer mehr Bürger leben am Rande des Existenzminimums. Trotz KVG und stetig ansteigender Ausgaben nehmen die Ungleichheiten zu. Beispiele aus den Kantonen Zürich und Genf bringen die Risikofaktoren an den Tag: tiefe Schulbildung, tiefes Einkommen, tiefer Sozialstatus, schlech- tes Arbeitsklima oder gar keine Arbeit. Die Prävalenz für Diabetes, Bronchitis, Hypertonie, Übergewicht, Gallensteine, Depressionen und Magengeschwüre ist bis vier Mal höher. Das gleiche Bild zeigt die Häufigkeitsverteilung von Invalidität und Mortalität infolge Krebs, psychischer Störungen oder Unfällen und Gewalt. Je niedriger die Position auf der sozialen Skala, desto kränker und behinderter sind die Menschen. Der Wohlstand verteilt sich zunehmend ungleich. Wirklich arm leben zwischen 10 und 15\% der Bevölkerung, während 3\% der Steuerpflichtigen über die Hälfte der Summe aller Nettovermögen verfügen (BFS 1999).

\section{Die alten und neuen Rezepte}

Mehrere Arbeitsgruppen suchen nach konkreten Strategien, wobei die Meinungen und Vorschläge stark auseinandergehen. Ein Konsens besteht darin, dass durch eine allgemeine Sensibilisierung für Gesundheitsfragen mehr soziale Gerechtigkeit resultieren könnte, dass Randgruppen noch gezielter durch Prävention aufzuklären seien und dass der Zugang zur Gesundheit als neuem Konsumgut in Zukunft noch mehr den sozialen Frieden bestimmen werde. Es fehlt nicht an Kommentaren und Rezepten. Sie reichen vom unermüdlich die Sozialziele der Verfassung zitierenden Chefbeamten, der Stolz verkündet, wie freundlich er seiner Putzfrau die Hand schüttelt, bis zur Gesundheitschefin, die sich fragt, was diese Tagung eigentlich soll. Ihre Kollegin aus dem Tessin preist dafür vehement die Errungenschaft ihrer Regierung: eine Gesundheitsverträglichkeitsprüfung für jedes neue Gesetz, es sei denn, es gehe um Steuern und Finanzen. Der Basler Departementschef provoziert am nächsten Tag, vor stark gelichteten Reihen, das versammelte BAG, indem er weitere Gesundheitsstudien und Befindlichkeitsumfragen für überflüssig erklärt, den wissenschaftlichen Ansatz der Projekte interessant, aber als Führungsprinzip nicht praktikabel und den Tessiner Vorschlag als technokratisch und die Exekutive behindernd qualifiziert. Zudem wolle die Bevölkerung nicht sparen, denn jeder wünsche für sich das Beste und das habe einen Preis, der sich im Rahmen üblicher Versicherungen bewege, was auch einmal klar in der Öffentlichkeit propagiert werden müsse. Was es brauche sei eine verbesserte Zusammenarbeit unter den Direktionen und eine gute Gesundheitserziehung in der Schule.

Zweimal fahre ich in der S-Bahn nach Rüschlikon und lese, dass die Zürcher Regierung die Steuern senken will und die Hälfte der Winterthurer Kindergärtner wieder an Karies leidet. Die real notwendige Prävention ist nicht spektakulär, sie ist die Sisyphusarbeit der immer gleichen kleinen Schritte. Daran werden die versprochenen zusätzlichen Indikatoren und weiteren Statistiken nichts ändern. Die helvetische Gesundheitspolitik hat hier keine Revolution angezettelt. Ihre Vertreter haben den weissen Kittel angezogen und der Gesellschaft ein bisschen den Puls gefühlt. 